This item was submitted to Loughborough's Research Repository by the author.

Items in Figshare are protected by copyright, with all rights reserved, unless otherwise indicated.

\title{
Dynamic characteristics in the design of MAGLEV suspensions
}

PLEASE CITE THE PUBLISHED VERSION

PUBLISHER

(C) IMechE / Professional Engineering Publishing

VERSION

VoR (Version of Record)

LICENCE

CC BY-NC-ND 4.0

REPOSITORY RECORD

Goodall, Roger M.. 2019. "Dynamic Characteristics in the Design of MAGLEV Suspensions". figshare. https://hdl.handle.net/2134/4695. 
This item was submitted to Loughborough's Institutional Repository (https://dspace.lboro.ac.uk/) by the author and is made available under the following Creative Commons Licence conditions.

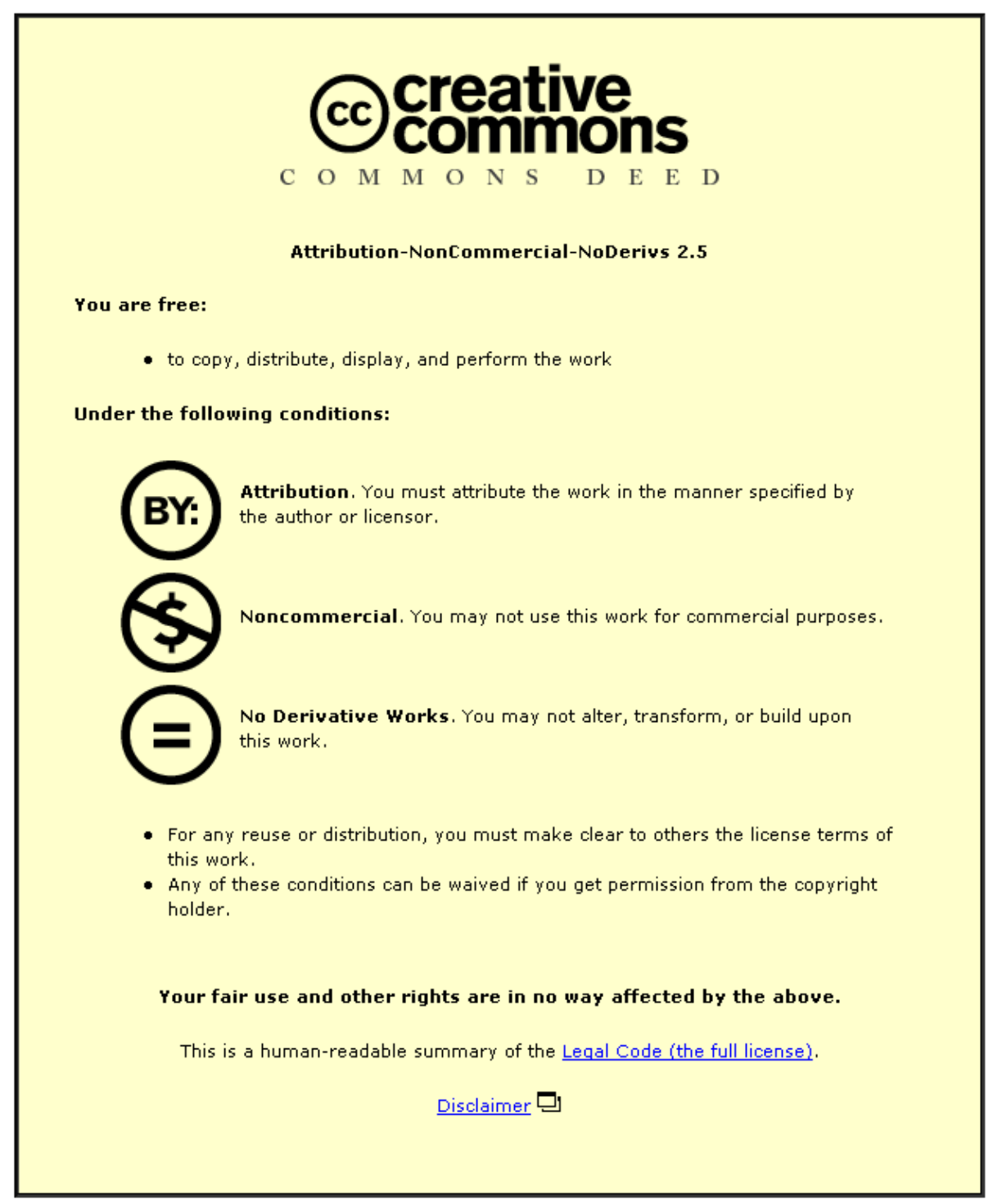

For the full text of this licence, please go to: http://creativecommons.org/licenses/by-nc-nd/2.5/ 


\section{Dynamic characteristics in the design of Maglev suspensions}

R Goodall, MA, PhD, CEng, FIEE, MInstMC

Department of Electronic and Electrical Engineering, Loughborough University of Technology

The paper reviews the essential functions which apply to any kind of suspension, and distinguishes between the various inputs to which a suspension is subjected. These are used to assess the particular characteristics of an electromagnetically suspended (Maglev) vehicle, and to identify considerations which have important implications for the controller design, irrespective of the design method. Some general equations are developed which interrelate the vehicle speed, the quality of the track and the passenger comfort requirements, and these are used to identify operational conditions for which a second stage of suspension becomes necessary (that is, in addition to that provided by the magnets). The importance of understanding the suspension's response to deterministic track inputs is also highlighted. Although the paper is directed towards Maglev, the analysis is strongly based upon a consideration of the suspension transfer functions, and so many of the principles are applicable to actively controlled supensions in general.

\section{NOTATION}

A track spectrum constant

$f_{0}, \omega_{0}$ cut-off frequency of suspension transfer function

$f_{\mathrm{t}} \quad$ spatial frequency

$g$ magnet airgap

$H(s)$ suspension transfer function

$K \quad$ suspension stiffness

$M \quad$ suspended mass

$V \quad$ vehicle speed along track

$z \quad$ vertical position of vehicle body

$z_{\mathrm{t}} \quad$ vertical position of track

$\delta \quad$ static deflection of suspension

\section{INTRODUCTION}

The suspension functions in a magnetic suspension are primarily provided by an all-electronic system, and it is tempting therefore to concentrate on the control aspects and forget the analogies with the passive (mechanical) suspension system that is being replaced. It is certainly true that active control of the suspension provided by the magnets (and other forms of actuator for active suspensions in general) enables the designer to incorporate features that are simply not possible with a normal suspension. However, it is important when designing the control system to identify clearly the functions of the suspension, its performance requirements and the constraints under which it must operate, issues which are often neglected by control engineers when they concentrate upon the control strategies.

This paper provides a link between the concepts with which suspension designers are familiar and the techniques used by control engineers, primarily by characterizing the suspension's response in terms of transfer functions. It defines clearly the full range of dynamic requirements for electromagnetic suspensions, and identifies key features which must be addressed by whatever approach is used for designing the control system. It also discusses operational limits based upon achieving specified levels of performance within the practical constraints of the technology.

The MS was recieved on 27 July 1993 and was accepted for publication on 22 March 1994
The analysis is mainly restricted to the suspension of a single mass by means of an electromagnet; the basic scheme is shown in Fig. 1, which also defines some of the important variables relating to the suspension. The assumption is that the fundamental instability of the Maglev suspension has been overcome. Many approaches which identify control laws for this purpose are available in the literature (1-3), and so the emphasis here is upon the characteristics of the closed-loop transfer function, irrespective of the particular control law used. The situation is more complicated for a real vehicle with four (or perhaps more) magnets, and consequently is less susceptible to a simple analysis by which trends and limits can be identified; the general conclusions nevertheless still apply. For a complete vehicle it is possible to achieve a performance which is slightly better than that predicted for a single mass (4), but the differences are not significant in the context of the broad assessment which is the subject of this paper.

The assessment is also restricted to the vertical suspension, since this is the most demanding because of the restricted airgap. The lateral suspension, however, must not be neglected with any guided vehicle because there will be important practical considerations to be dealt with, depending upon the configuration of the magnetic suspension, and many of the issues which are

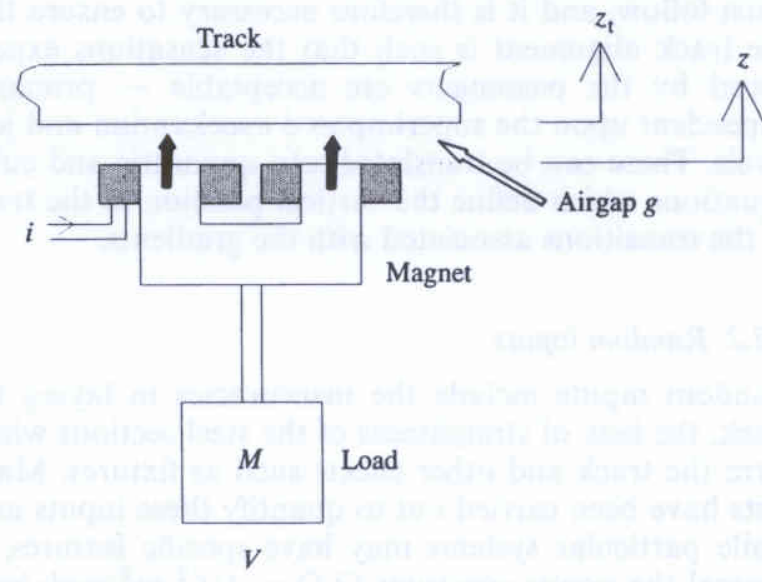

Fig. 1 Basic suspension arrangement 
identified for the vertical direction also apply to the lateral suspension.

\section{SUSPENSION BASICS}

The purpose of this section is to clarify the functions of a suspension and the inputs to which it is subjected, to explain what is expected in the way of performance and to identify the design constraints.

\subsection{Functions of a suspension}

The three primary functions of a suspension are as follows:

(a) to support the payload with respect to the track;

(b) to follow the intended variations in the position of the track;

(c) to provide isolation from the unintentional irregularities in the track position.

There is a sense in which (a) and (b) are similar functions, but they are listed separately because they help to identify distinct design issues. Notice that some of these functions are in the limit mutually exclusive, which highlights the need for compromise in any suspension design.

\subsection{Inputs to a suspension}

A suspension system is subjected to a variety of inputs, which may be subdivided into three types: deterministic track inputs representing specific features such as curves and gradients, random track inputs representing the uncertainty compared with the track's intended position and also the force inputs which must be reacted by the suspension.

\subsubsection{Deterministic inputs}

These depend upon the routing requirements for the particular transport system and the terrain over which it must cross, and the track will be defined geometrically to accommodate specific performance requirements. If improperly specified the intended variations in the track input will cause discomfort to passengers, and for the vertical direction these will be associated with running on and off gradients. They are clearly lowfrequency/long-wavelength features which the vehicle must follow, and it is therefore necessary to ensure that the track alignment is such that the sensations experienced by the passengers are acceptable - primarily dependent upon the superimposed acceleration and jerk levels. These can be translated into quadratic and cubic equations which define the vertical position of the track at the transitions associated with the gradients.

\subsubsection{Random inputs}

Random inputs include the inaccuracies in laying the track, the lack of straightness of the steel sections which form the track and other effects such as fixtures. Many tests have been carried out to quantify these inputs and, while particular systems may have specific features, in general the power spectrum $G\left(f_{t}\right)=A / f_{\mathrm{t}}^{2} \mathrm{~m}^{2} /$ cycle $/ \mathrm{m}$ is broadly representative of the vertical alignment of the track, where $f_{\mathrm{t}}$ cycle $/ \mathrm{m}$ is the spatial frequency and $A$ is a track roughness factor. [This is a simplification because for track wavelengths shorter than around $5 \mathrm{~m}$ the spectrum is reduced (5), but the approximation serves the purpose of this paper.] The spectral shape is substantially constant over a range of track qualities, although the value of $A$ changes; $A=10^{-6}$ is commonly taken to represent secondary quality track, and $A=2.5 \times 10^{-7}$ for best-quality high-speed track. (In the latter case the standard deviation over a $50 \mathrm{~m}$ length is in the region of $2 \mathrm{~mm}$.)

Converting the spectrum to use temporal frequency (that is in $\mathrm{Hz}$ ) involves the vehicle speed $V$, and can be shown to be $G^{\prime}(f)=A V / f^{2} \mathrm{~m}^{2} / \mathrm{Hz}$. It is interesting to represent this in terms of track velocity, for which the spectrum is $G_{v}^{\prime}(f)=(2 \pi)^{2} A V(\mathrm{~m} / \mathrm{s})^{2} / \mathrm{Hz}$, that is a flat power spectrum, the amplitude of which is proportional to the vehicle speed.

\subsubsection{Force inputs}

The other important inputs are the forces to which the suspension must react. Such forces are either changes in payload or external disturbances such as aerodynamic forces, wind, braking loads, etc. The character of the load variations will depend upon the application. There will be significant payload variations from fully laden to empty for both low- and high-speed systems. The changes will be larger for low-speed vehicles because they are usually of a lightweight construction, and will typically be $35-40$ per cent for low speed and $20-25$ per cent for high speed. They will also be more rapid, because it may only take a matter of seconds for a small low-speed vehicle to empty, whereas emptying a large high-speed passenger coach will usually take at least a minute.

\subsection{Performance requirements}

The primary requirement of a suspension is the quality of ride which it must deliver. This is an indication of its effectiveness in providing isolation from the track's roughness, and is quantified in terms of the r.m.s. acceleration experienced by the passengers. The r.m.s. acceleration level is determined from the acceleration spectrum, predicted from a combination of the transfer function of the vehicle's suspension and the track input spectrum. The spectrum should be 'weighted' as a function of frequency in order to allow for human susceptibility to vibration at different frequencies (6), but this weighting has not been incorporated because the assessment is deliberately broad to give overall design guidelines - it would of course be necessary to consider weighted accelerations for a particular suspension design. The acceptable level of r.m.s. acceleration depends upon the journey time, and commonly accepted criteria are $0.45 \mathrm{~m} / \mathrm{s}^{2}$ for the relatively short journeys on a low speed transport system, and 0.2 $\mathrm{m} / \mathrm{s}^{2}$ for longer journeys on high-speed inter-city transport. (The figures quoted are for vertical acceleration-somewhat lower levels are specified for the lateral direction, but these are not considered here.)

Requirements for the deterministic track inputs must also be specified, as discussed in Section 2.2. There is a difference between low- and high-speed systems, with acceleration levels often specified to be $2 \mathrm{~m} / \mathrm{s}^{2}$ and 1 


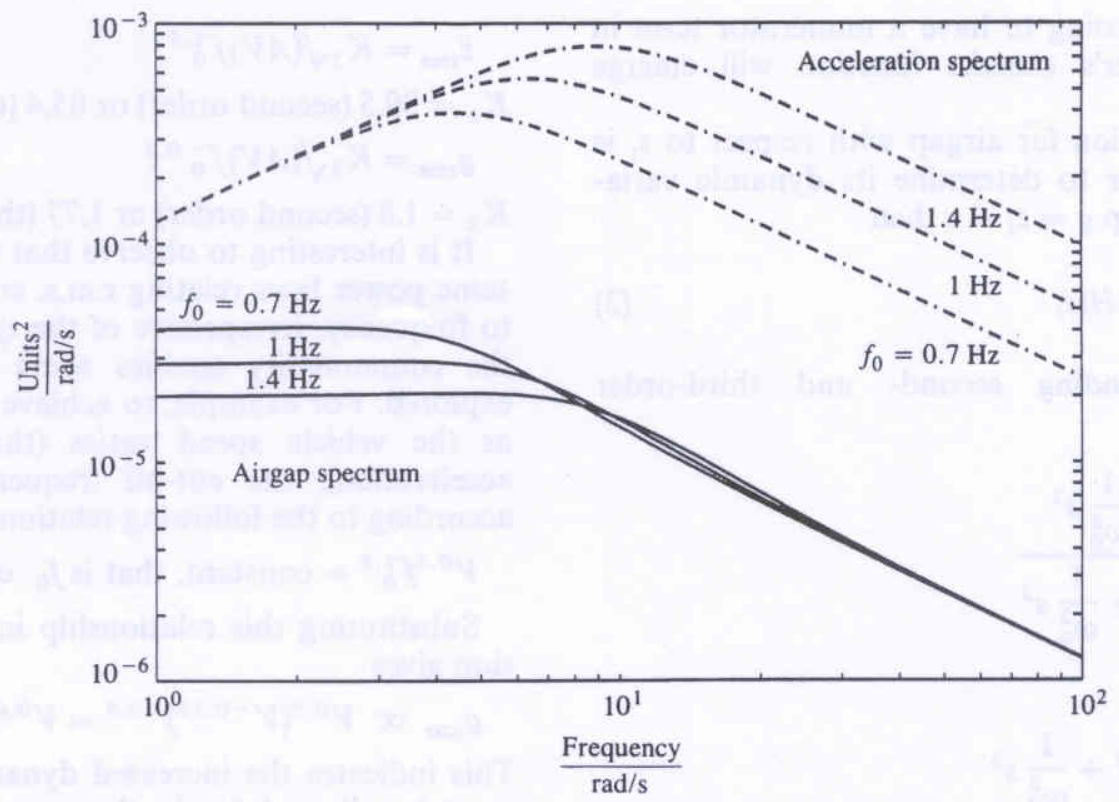

Fig. 2 Effect of suspension frequency on acceleration and airgap spectra (second-order response)

$\mathrm{m} / \mathrm{s}^{2}$ respectively, with the corresponding jerk levels as $1.25 \mathrm{~m} / \mathrm{s}^{3}$ and $0.5 \mathrm{~m} / \mathrm{s}^{3}$ (7), although on many highspeed routes the vertical jerk limit is not incorporated and an acceleration limit of $0.5 \mathrm{~m} / \mathrm{s}^{2}$ is used. Note that the track spectrum mentioned previously will not adequately describe these inputs, and so it is necessary to check independently the suspension's response as the vehicle negotiates gradients.

The fundamental constraint within which these performance requirements must be achieved is the maximum allowable displacement of the suspension. For a Maglev vehicle this is governed by the size of the airgap, for which $15 \mathrm{~mm}$ is often chosen, and in some cases less. It is of course possible to use a larger airgap, but the magnetic circuit becomes most unfavourable and so most workers have avoided this trend. Isolating the passengers from the track roughness means that there must be dynamic variations in the airgap; the provision of this isolation in turn means that negotiating gradients will inevitably cause airgap changes, and together these effects must not compromise the ride quality by too frequent contact with the track. On passive suspensions the maximum suspension movement is usually specified to be five times the r.m.s. gap change, but with an actively controlled suspension a figure of perhaps three or four times can be used because it is possible to 'stiffen' the suspension electronically just prior to contact. For Maglev therefore the r.m.s. variations in the airgap must be restricted to 4 or $5 \mathrm{~mm}$, and transient changes at gradients to a similar level. Any deflections of the suspension caused by the various force inputs are also constrained by the airgap. Strictly, the three contributions to airgap changes should be rigorously combined, although in practice, as long as they are each taken sensibly into account, a separate approach is found to give a satisfactory performance.

\section{SUSPENSION DESIGN}

Although the various aspects of a suspension must in practice be considered in parallel, they are presented here in sequence because this helps to develop the essential underlying principles. First to be considered is the response to random track inputs, then the response to deterministic track inputs, and then to load changes.

\subsection{Response to random track inputs}

A suspension may be thought of as a low-pass filter, with only the long-wavelength low-frequency track variations being passed on to the vehicle. In a passive suspension the springs, dampers, masses, etc., determine the filter's characteristics, although in practice the type of filter response so caused is quite restricted. In an active suspension the transfer function can be electronically defined, and, while there are some practical limitations to what can be achieved, the addition of active control opens up completely new possibilities. Second- and third-order transfer functions with varying cut-off frequencies are used here to represent the effect of a Maglev suspension. Butterworth coefficients are chosen because they are known to give a good general purpose filter characteristic, and also because previous work (8) has shown this to be the optimum for a second-order suspension response.

If the basic transfer function relating the vertical position $z$ of the mass to that of the track $z_{t}$ is

$$
\frac{z(s)}{z_{t}(s)}=H(s)
$$

then the second- and third-order transfer functions, using Butterworth coefficients, are

$$
H_{2}(s)=\frac{1}{1+\frac{\sqrt{2}}{\omega_{0}} s+\frac{1}{\omega_{0}^{2}} s^{2}}
$$

or

$$
H_{3}(s)=\frac{1+\frac{2}{\omega_{0}} s}{1+\frac{2}{\omega_{0}} s+\frac{2}{\omega_{0}^{2}} s^{2}+\frac{1}{\omega_{0}^{3}} s^{3}}
$$


(The reason for choosing to have a numerator term in the third-order filter's transfer function will emerge later.)

The transfer function for airgap with respect to $z_{t}$ is also needed in order to determine its dynamic variations. Since the airgap $g=z_{\mathrm{t}}-z$ then

$$
\frac{g(s)}{z_{\mathrm{t}}(s)}=H^{\prime}(s)=1-H(s)
$$

with the corresponding second- and third-order responses being

$$
H_{2}^{\prime}(s)=\frac{\frac{\sqrt{ } 2}{\omega_{0}} s+\frac{1}{\omega_{0}^{2}} s^{2}}{1+\frac{\sqrt{2}}{\omega_{0}} s+\frac{1}{\omega_{0}^{2}} s^{2}}
$$

or

$$
H_{3}^{\prime}(s)=\frac{\frac{2}{\omega_{0}^{2}} s^{2}+\frac{1}{\omega_{0}^{3}} s^{3}}{1+\frac{2}{\omega_{0}} s+\frac{2}{\omega_{0}^{2}} s^{2}+\frac{1}{\omega_{0}^{3}} s^{3}}
$$

Figure 2 shows the spectra of acceleration and airgap change for the random track input for the second-order transfer function with cut-off frequencies of 0.7, 1.0 and $1.4 \mathrm{~Hz}$. At low frequencies the acceleration spectra are all the same, which corresponds to the vehicle following the track. At high frequencies the airgap spectra are all the same, which is where the suspension is providing isolation and all the track movement appears in the airgap. It can be seen that a lower cut-off frequency decreases the acceleration spectrum but increases the airgap spectra, and this indicates the essential design conflict between ride quality and suspension displacement.

The r.m.s. acceleration can then be derived as follows:

$$
\ddot{z}_{\mathrm{rms}}=\sqrt{\left\{\int_{0}^{\infty} \frac{A V}{f^{2}}\left[(j \omega)^{2} H(j \omega)\right]\left[(-j \omega)^{2} H(-j \omega)\right] \mathrm{d} f\right\}}
$$

A similar derivation will give the r.m.s. change in airgap

$$
g_{\mathrm{rms}}=\sqrt{ }\left\{\int_{0}^{\infty} \frac{A V}{f^{2}}\left[H^{\prime}(j \omega)\right]\left[H^{\prime}(-j \omega)\right] \mathrm{d} f\right\}
$$

(Note that both expressions are based upon a singlesided spectrum.)

These integrals can readily be evaluated to give the expressions for the r.m.s. acceleration and suspension deflection, which are shown below for both second- and third-order filters, in which $f_{0}=\omega_{0} / 2 \pi$ is the filter cut-off frequency in $\mathrm{Hz}$.

$$
\ddot{z}_{\text {rms }}=K_{1} \sqrt{ }(A V) f_{0}^{1.5}
$$

$K_{1}=39.5$ (second order) or 85.4 (third order).

$$
g_{\mathrm{rms}}=K_{2} \sqrt{ }(A V) f_{0}^{-0.5}
$$

$K_{2}=1.8$ (second order) or 1.77 (third order).

It is interesting to observe that the analysis yields the same power laws relating r.m.s. acceleration and airgap to frequency, irrespective of the type of filter used, and the commonality enables some of the trends to be explored. For example, to achieve the same ride quality as the vehicle speed varies (that is constant r.m.s. acceleration), the cut-off frequency must be altered according to the following relationship:

$$
V^{0.5} f_{0}^{1.5}=\text { constant, that is } f_{0} \propto V^{-0.33}
$$

Substituting this relationship into the airgap expression gives

$$
g_{\text {rms }} \propto V^{0.5}\left(V^{-0.33}\right)^{-0.5}=V^{0.67}
$$

This indicates the increased dynamic movement which must be allowed for in the suspension to achieve the same ride quality as speed is increased, assuming the same quality of track.

In fact it is not as simple as this, because as mentioned earlier higher speed is generally associated with longer journey times, requiring lower levels of r.m.s. acceleration, and so there will be a compounding effect, which means that the airgap changes (that is, suspension deflection) will rise more rapidly with speed than equation (10) suggests. Also, higher speed track will be of higher quality, but the trends indicated by these expressions are important to appreciate. A later section suggests the design flow by which the equations can be used for a particular design.

Alternatively, an expression can be derived which indicates the way in which the r.m.s. acceleration will vary with speed on the basis of achieving a given airgap requirement. From equation (8), constant $g_{\text {rms }}$ means that $f_{0} \propto V$, and substituting into equation (7) yields the relationship:

$$
\ddot{z}_{\text {rms }} \propto V^{2}
$$

Table 1 summarizes some of the results which can be derived from this analysis. For a low-speed system with typical speeds of around $15 \mathrm{~m} / \mathrm{s}$ running on secondary quality track $\left(A=10^{-6}\right)$, a $2 \mathrm{~Hz}$ second-order filter is needed to keep the r.m.s. acceleration below $0.45 \mathrm{~m} / \mathrm{s}^{2}$;

\begin{tabular}{|c|c|c|c|c|c|}
\hline \multirow[b]{2}{*}{ Application } & \multirow[b]{2}{*}{ Track quality } & \multicolumn{2}{|c|}{ Filter } & \multirow{2}{*}{$\begin{array}{c}\text { r.m.s. } \\
\text { acceleration } \\
\left(\mathrm{m} / \mathrm{s}^{2}\right)\end{array}$} & \multirow{2}{*}{$\begin{array}{l}\text { r.m.s. } \\
\text { airgap } \\
(\mathrm{mm})\end{array}$} \\
\hline & & Type & $f_{0}(\mathrm{~Hz})$ & & \\
\hline $\begin{array}{l}\text { Low speed } \\
(20 \mathrm{~m} / \mathrm{s})\end{array}$ & $\begin{array}{l}\text { Second class } \\
\qquad\left(A=10^{-6}\right)\end{array}$ & $\begin{array}{l}\text { Second } \\
\text { Third }\end{array}$ & $\begin{array}{l}2 \\
1.2\end{array}$ & $\begin{array}{l}0.45 \\
0.45\end{array}$ & $\begin{array}{l}4.9 \\
6.2\end{array}$ \\
\hline $\begin{array}{l}\text { High speed } \\
(45 \mathrm{~m} / \mathrm{s})\end{array}$ & $\begin{array}{l}\text { First class } \\
\qquad\left(A=2.5 \times 10^{-7}\right)\end{array}$ & $\begin{array}{l}\text { Second } \\
\text { Third }\end{array}$ & $\begin{array}{l}1.4 \\
0.84\end{array}$ & $\begin{array}{l}0.22 \\
0.22\end{array}$ & $\begin{array}{l}5 \\
6.5\end{array}$ \\
\hline
\end{tabular}
the corresponding change in airgap is $4.9 \mathrm{~mm}$ r.m.s., which is just acceptable. To achieve the same ride quality with a third-order filter requires a cut-off frequency of $1.2 \mathrm{~Hz}$, but the r.m.s. change in the airgap increases to $6.2 \mathrm{~mm}$, which is obviously becoming excessive, and so the second-order filter gives a better design trade-off for random track inputs. With a higher quality of track considerable improvements in performance are

Table 1 R.m.s. suspension parameters under different operational conditions 
possible, but this would not be normal for a low-speed system because of the extra cost of alignment and maintenance. It is worth remarking that, for a conventional vehicle with a passive suspension, the ride quality would be achieved with a lower natural frequency for the suspension, taking advantage of the extra suspension deflection which is normally available when using springs, dampers, etc.

For a high-speed system, for which the r.m.s. acceleration needs to be significantly reduced, as mentioned in Section 2.3, the required performance within the airgap constraint can only be achieved through improved track quality. For first-class track $\left(A=2.5 \times 10^{-7}\right)$ a speed of $45 \mathrm{~m} / \mathrm{s}$ can be achieved with a $1.4 \mathrm{~Hz}$ secondorder filter (giving r.m.s. values of $0.22 \mathrm{~m} / \mathrm{s}^{2}$ and $5 \mathrm{~mm}$ ) or with a $0.8 \mathrm{~Hz}$ third-order filter $\left(0.22 \mathrm{~m} / \mathrm{s}^{2}\right.$ and $\left.6 \mathrm{~mm}\right)$. In principle, therefore, the improved suspension performance provided by active control can enable higher speed operation with a single stage of suspension, but only on high-quality track, and with the restricted airgap the kind of speed which is now considered as high (that is greater than $55 \mathrm{~m} / \mathrm{s}$ ) is not possible. It will emerge, however, that there are other factors which also mean that high-speed operation is not feasible.

\subsection{Response to deterministic inputs}

As mentioned earlier, although the vehicle will fundamentally be following the long-wavelength variations in the track position, there must nevertheless be some dynamic changes in the airgap in order to provide isolation from short-wavelength variations, and these can be derived from the $g / z_{\mathrm{t}}$ transfer function. For example, for second- and third-order filters

$$
g(s)=\frac{\frac{\sqrt{ } 2}{\omega_{0}} s+\frac{1}{\omega_{0}^{2}} s^{2}}{1+\frac{\sqrt{2}}{\omega_{0}} s+\frac{1}{\omega_{0}^{2}} s^{2}} z_{\mathrm{t}}(s)
$$

or

$$
g(s)=\frac{\frac{2}{\omega_{0}^{2}} s^{2}+\frac{1}{\omega_{0}^{3}} s^{3}}{1+\frac{2}{\omega_{0}} s+\frac{2}{\omega_{0}^{2}} s^{2}+\frac{1}{\omega_{0}^{3}} s^{3}} z_{t}(s)
$$

For a specific deterministic track input it would be possible to calculate the exact response, but a more general approach can also be used to assess directly the quasi steady-state airgap changes corresponding to a particular suspension transfer function. It has already been stressed that the deterministic features of the track are effectively low-frequency inputs. They will generally be sustained for a second or so, whereas the time constant for the suspension's transient response using the cut-off frequencies derived in Section 3.1 will typically be around a tenth of a second. Consequently, the tran- sient response to the velocity, acceleration and jerk inputs will die away relatively quickly, and the steadystate suspension deflection with second- and third-order filters can therefore be seen from the numerators of the transfer functions to be:

(a) Second order:

$$
g_{\mathrm{ss}}=\frac{\sqrt{2}}{\omega_{0}} \dot{z}_{\mathrm{t}}+\frac{1}{\omega_{0}^{2}} \ddot{z}_{\mathrm{t}}
$$

(b) Third order:

$$
g_{\mathrm{ss}}=\frac{2}{\omega_{0}^{2}} \ddot{z}_{\mathrm{t}}+\frac{1}{\omega_{0}^{3}} \dddot{z}_{\mathrm{t}}
$$

Notice that, whereas the second-order response has a term proportional to the track velocity, the third-order response does not. This is a consequence of the particular form of third-order transfer function, chosen to have a term in the numerator, the effect of which will become clear in the following paragraphs.

A value for the vertical velocity of the track corresponding to the deterministic inputs is needed to calculate the deflections with the second-order filter. This was not specified when maximum levels were being discussed in Section 2.3 because velocity itself is unperceived by passengers, and therefore not associated with discomfort. Maximum gradients for low- and highspeed systems are likely to be around 10 per cent and 3 per cent respectively; at corresponding speeds of $15 \mathrm{~m} / \mathrm{s}$ and $75 \mathrm{~m} / \mathrm{s}$ the vertical velocities therefore work out at $1.5 \mathrm{~m} / \mathrm{s}$ and $2.25 \mathrm{~m} / \mathrm{s}$.

Table 2 gives examples of the suspension deflections which can be calculated using this approach, as well as the corresponding filter characteristics and the maximum velocity, acceleration and jerk levels. Note that the filter frequencies are those derived in Section 3.1 when considering the response to random inputs.

The very large deflections shown in this table underline how important it is to consider deterministic inputs as well as random inputs when designing a suspension's characteristics. Fortunately, there are techniques which will reduce these deflections, essentially by modifying the low-frequency response of the filters which are used to determine the suspension's transfer functions, although this will inevitably deteriorate the ride quality compared with the figures given earlier in the paper. Even so it is quite difficult to reduce the large deflections created by the second-order filter to acceptable levels (the mechanical analogy being a 'skyhook' damper connected to an absolute datum), and this is the reason for the particular form of third-order filter introduced in Section 3.1. The term in the numerator avoids this type of damping as equations (12) and (13) show, but of course the ability to attenuate the random inputs

\begin{tabular}{|c|c|c|c|c|c|c|c|}
\hline \multirow[b]{2}{*}{ Application } & \multicolumn{3}{|c|}{ Maxima } & \multicolumn{2}{|c|}{ Second order } & \multicolumn{2}{|c|}{ Third order } \\
\hline & $\begin{array}{l}\text { Velocity } \\
(\mathrm{m} / \mathrm{s})\end{array}$ & $\begin{array}{l}\text { Acceleration } \\
\left(\mathrm{m} / \mathrm{s}^{2}\right)\end{array}$ & $\begin{array}{c}\text { Jerk } \\
\left(\mathrm{m} / \mathrm{s}^{3}\right)\end{array}$ & $\begin{array}{c}\text { Frequency } \\
(\mathbf{H z})\end{array}$ & $\begin{array}{l}\text { Deflection } \\
\text { (mm) }\end{array}$ & $\begin{array}{c}\text { Frequency } \\
(\mathrm{Hz})\end{array}$ & $\begin{array}{l}\text { Deflection } \\
\text { (mm) }\end{array}$ \\
\hline Low speed & 1.5 & 2 & 1.25 & 2 & 181 & 1.2 & 73 \\
\hline High speed & 2.25 & 1 & 0.5 & 1.4 & 326 & 0.84 & 53 \\
\hline
\end{tabular}
within the constraint of the airgap is significantly degraded, as demonstrated by the results in Table 1 .

Table 2 Suspension deflections with deterministic inputs 
The figures used in the analysis are very much the worst case. For specific applications, much reduced levels of velocity, acceleration and jerk might be applicable, in which case it may be possible to take advantage of the second-order response, because there is no doubt that if it is possible to use a second-order transfer function this gives a much superior ride quality. The purpose has been, however, to highlight the importance of the deterministic inputs and the difficulties of accommodating their effects within the restricted suspension travel of a Maglev system, issues which are rarely addressed in the literature, although the problems with 'skyhook damping' are starting to become recognized in considering active suspensions for automobiles (9).

\subsection{Response to force inputs}

In a passive suspension there is a clear link between the natural frequency $f_{0}$ and static deflection $\delta$ caused by the gravitational force on the suspended mass $M$ acting on the spring stiffness $K$ :

$$
f_{0}=\frac{1}{2 \pi} \sqrt{\frac{K}{M}}
$$

and

$$
K=\frac{M g}{\delta}
$$

Hence

$$
\delta \approx \frac{0.25}{f_{0}^{2}}
$$

For a $1 \mathrm{~Hz}$ suspension for example, the static deflection is $250 \mathrm{~mm}$. Obviously it is normal to preload the suspension, but it is still necessary to accommodate load changes. Even with the relatively large movements available with a passive suspension this has always been recognized as a difficulty in achieving low suspension frequencies, and has been tackled with self-levelling suspensions using airsprings, which have the additional advantage of helping to keep the suspension frequency relatively constant as the load changes.

For a Maglev suspension, with its more restricted suspension movement, it is essential that the response to load changes is properly allowed for because it is clearly unacceptable to have load deflections of this sort, even with the reduced changes which would be encountered as the load varies from empty to fully laden. For a lowspeed vehicle having a suspension frequency of $1.2 \mathrm{~Hz}$ (third-order filter) and load changes of around \pm 20 per cent the corresponding load deflection works out at $\pm 35 \mathrm{~mm}$; for a high-speed vehicle $(0.84 \mathrm{~Hz}$ suspension and \pm 12 per cent load variation) it becomes $\pm 42 \mathrm{~mm}$. Fortunately, unlike a passive suspension, active control provides the opportunity to react to forces in a way which is not linked to the suspension frequency. For example, if acceleration measurements are used directly to give the dynamic characteristics of the suspension, force inputs will be reacted to very rapidly, but acceleration feedback can cause practical difficulties because of resonant modes in the magnet supports or the vehicle structure. Alternative methods exist which do not cause such problems (for example, see 3 ), but the point being stressed here is that the effect of load variation must be properly addressed or else the vehicle will be very susceptible to shifting passenger loads or to wind buffeting, both of which must be properly accommodated to ensure robust operation of a transportation system.

\subsection{Other considerations}

The preceding subsection has identified some of the difficulties of achieving high speeds with a single stage of suspension. Although the intention has been to concentrate upon suspension principles applied to a single magnet and load, there is one aspect related to a complete vehicle which imposes a further severe constraint upon the use of a single-stage suspension for high-speed operation - that is the problem of track twist. At high speeds the track will be canted or banked around curves to increase the speed through the curve without imposing excessive lateral acceleration upon the passengers. At $60 \mathrm{~m} / \mathrm{s}$ the transition onto the canted section will be around $90 \mathrm{~m}$ long; the lateral acceleration then rises to its maximum level over $1.5 \mathrm{~s}$, which is typically what is dictated by passenger comfort requirements in the lateral direction. At the end of the transition the outer rail will be in the region of $150 \mathrm{~mm}$ higher than the inner rail, giving a 'track twist' of $1.7 \mathrm{~mm}$ per $\mathrm{m}$ along the track during the transition. A high-speed vehicle will usually be about $25 \mathrm{~m}$ long, so with a single stage of suspension the longitudinal spacing between front and back magnets will be in the region of $18 \mathrm{~m}$. On the transition to curves there will be a track twist over this length of a little over $30 \mathrm{~mm}$, clearly impossible with $15 \mathrm{~mm}$ airgaps.

In practice, therefore, the upper speed limit for a single-stage suspension is likely to be $40-50 \mathrm{~m} / \mathrm{s}$, beyond which it is necessary to use a separate secondary suspension, either using a configuration with bogies somewhat similar to a conventional railway suspension or the 'magnetic wheel' concept pioneered in the German Transrapid vehicles (10) in which the magnets are distributed along the length of the vehicle.

\section{TWO-STAGE SUSPENSIONS}

If the magnets are fitted directly to a bogie, with a connection to the vehicle body via a soft secondary stage of suspension (passive or active), a number of the problems identified above for high-speed operation are avoided. The primary magnetic suspension can now be relatively stiff, which means that dynamic changes in airgap are reduced for both random and deterministic track inputs; also, the much closer longitudinal spacing of adjacent magnets on each bogie means that track twist can be easily accommodated within the restricted airgap. The stiffer suspension of course means that the acceleration levels on the bogie will be much increased, and it is important to ensure that the control of the magnet excitation is sufficiently fast and large to provide these accelerations. For example, a $7 \mathrm{~Hz}$ suspension on secondary quality track at $75 \mathrm{~m} / \mathrm{s}$ will cause $6 \mathrm{~m} / \mathrm{s}^{2}$ r.m.s. acceleration at the magnets with about $6 \mathrm{~mm}$ r.m.s. change in the airgap. At first sight the peak accelerations of well over 1 ' $g$ ' appear to be a problem. 
(Whole vehicle mass $=M$ )

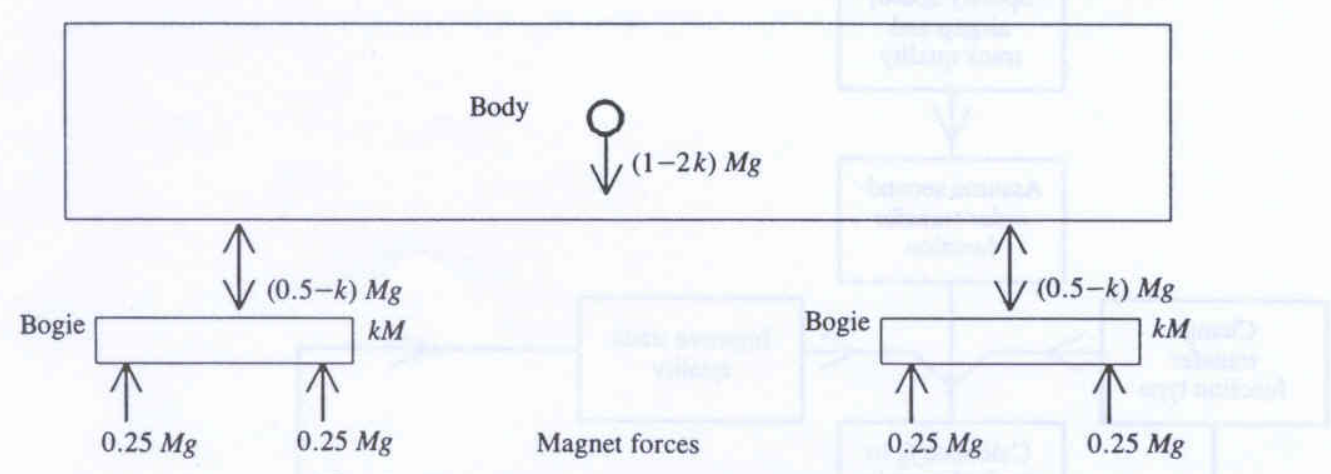

Fig. 3 Two-stage suspension arrangement

However, consideration of Fig. 3 shows the steady-state forces in the vertical direction. With each bogie being a fraction $k$ of the total vehicle mass, the maximum downward acceleration of the bogie occurs when the magnet forces are zero, that is

$$
\ddot{z}_{\max }=\frac{(0.5-k)}{k} g \mathrm{~m} / \mathrm{s}^{2}
$$

To achieve an equal upwards bogie acceleration each magnet must generate a maximum force of

$$
F_{\max }=0.25 M g+\frac{(0.5-k)}{k} \frac{k M}{2} g
$$

which can readily be expressed as of factor of $(2-2 k)$ times the static force level of $0.25 \mathrm{Mg}$.

For a typical vehicle in which the bogies account for around 30 per cent of the total weight $(k=0.15)$, the maximum downwards acceleration of the bogies will be in excess of $20 \mathrm{~m} / \mathrm{s}^{2}$, which clearly makes the previous example requiring an r.m.s. acceleration level of $6 \mathrm{~m} / \mathrm{s}^{2}$ more achievable.

It will, however, be necessary to ensure that a significant increase in force can be achieved transiently - a 70 per cent increase for the numerical example given above. This has an impact upon the magnet design and will probably result in an increased weight because the working flux density must be reduced so that the maximum force can be achieved without saturating the magnets. Note that these enhanced force levels are not needed for a single-stage suspension, for which the magnet can therefore be designed reasonably close to saturation.

The two-stage suspension therefore solves some of the problems, but of course the essential simplicity of a single-stage electromagnetic suspension is lost, and represents one of the costs of high-speed transportation.

\section{OUTLINE DESIGN METHODOLOGY}

In order to bring together the various requirements which have been mentioned, Fig. 4 presents the design flow which identifies the important suspension design decisions.

The first stage is to define the essential operational characteristics: speed, track quality and working airgap for the magnets. Since it is clear that a second-order transfer function is to be preferred if possible, the next stage is to calculate the cut-off frequency and decide whether the ride quality is likely to be adequate. If it proves to be good enough it is then necessary to check whether there will be any problem with the deterministic inputs, and if necessary change the type of transfer function. If the ride quality proves to be inadequate, assuming that the airgap cannot be increased, there are two choices: either improve the track quality or add a second stage of suspension.

In the case of a secondary suspension an estimate must be made of the likely bogie weight (based upon the weight of magnets and their supporting structure). Having chosen an appropriate cut-off frequency for the primary magnetic suspension in order to satisfy the airgap requirement, it is then necessary to determine whether the required maximum acceleration levels can be achieved. If not, the track quality must be improved. Having made the change to a two-stage suspension it may prove easy to achieve the required ride quality, in which case consideration can be given either to decreasing the operating airgap of the magnet or to using a lower track quality. No consideration has been given here to designing the secondary suspension, which is a more complex process involving a number of factors, including of course the dynamic characteristics of the magnetic primary suspension.

There are of course many other issues which must be dealt with, but the procedure described will be sufficient to take some of the important 'top level' decisions about the suspension configuration.

\section{CONTROL CONSIDERATIONS}

The previous sections have identified the performance requirements and characteristics for the controlled suspension. A number of design approaches can be used to achieve this, and both classical and optimal techniques have been successfully employed. Optimal techniques are well suited of course to satisfying the requirements for the random track inputs, although the other aspects of suspension design need more careful consideration.

At this stage it has been assumed that the magnets can be controlled in such a way as to give whatever suspension transfer function is required. The reality is fairly close to the ideal because electromagnets offer high bandwidth actuation. The non-contacting nature of their action means that non-linearities such as friction and backlash do not exist. There is some hysteresis 


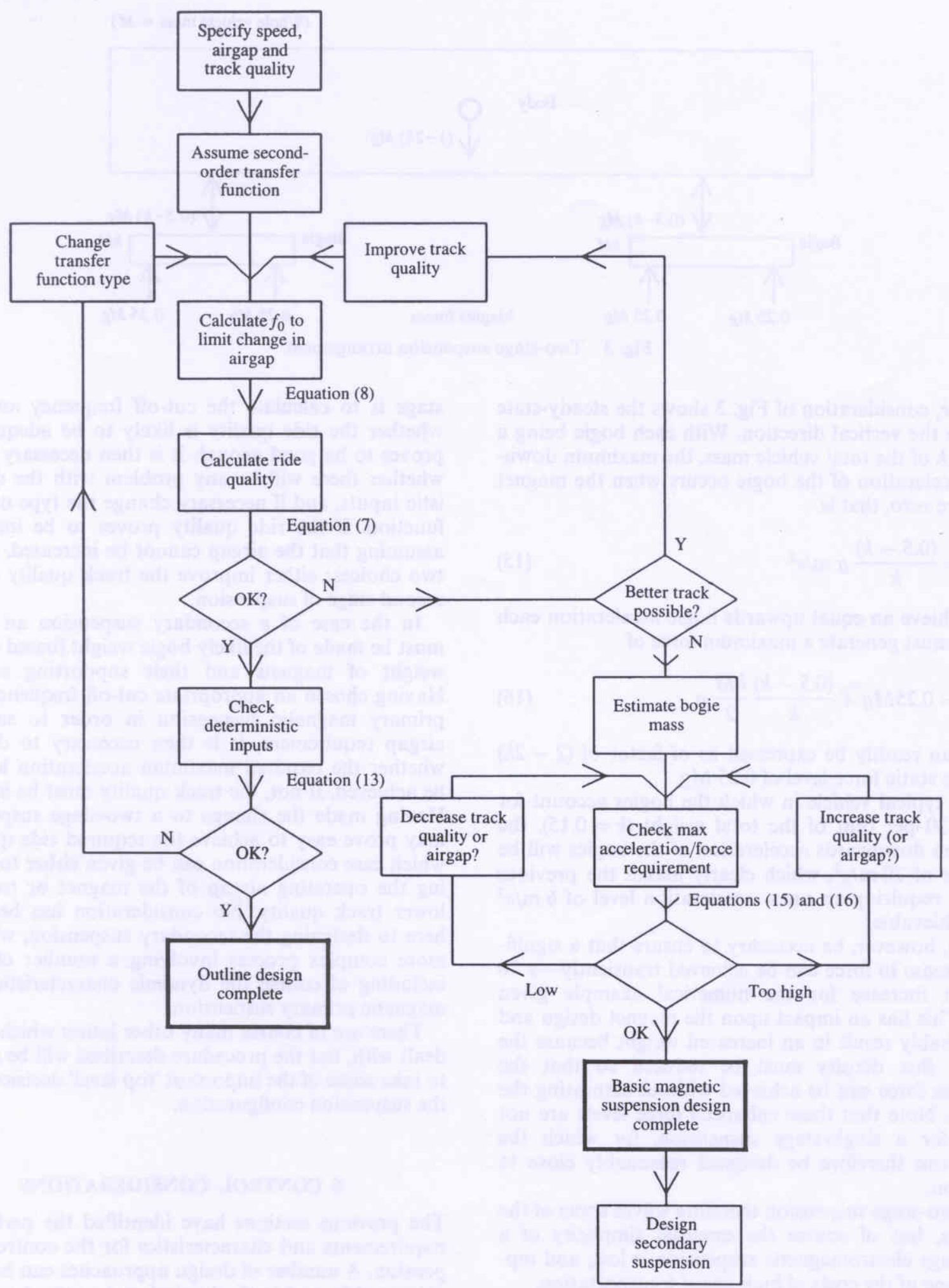

Fig. 4 Design flow diagram

but its effect is not large. The basic equations of the magnet are certainly non-linear, but the non-linearities are 'soft' and in general do not cause much problem in controllability. An essential factor related to controllability is to ensure that the magnet excitation is sufficient. Its current is primarily determined by the force requirement, comments regarding which were made in Section 4, but it is also important that there is sufficient voltage to force the changes in current. For a single- stage suspension the overall aim is to keep the dynamic variation in force small (and hence in flux density) in order to provide a good ride quality, and it is interesting to observe that the basic principle of electromagnetic induction is consistent with this aim, which means that the inductive voltage requirements are relatively small. For a high-speed two-stage suspension the situation is quite different however. Not only will large dynamic variations in force be needed for the magnets 
to follow the track, but also the frequency at which such variations are needed will be significantly higher, and the power amplifiers used to control the magnets must be carefully specified if effective control is to be achieved under all operating conditions.

\section{CONCLUSION}

The paper has endeavoured to highlight the important dynamic characteristics for electromagnetic suspensions. Suspension design in general is a classic example of achieving a satisfactory trade-off between conflicting requirements, and this is where the challenge of Maglev lies. Despite the fundamental instability and the 'magic' of non-contacting levitation, simply to achieve stable levitation is relatively straightforward, especially since an electromagnet contains little in the way of problematic non-linearities. In the author's experience the difficulties lie in designing a robust and cost-effective control system which satisfies all the suspension requirements simultaneously. This paper has highlighted the key implications, and has endeavoured to show how these might be linked together when designing a Maglev suspension, in particular to identify whether a second stage of suspension is necessary.

Many of the requirements and design constraints apply equally to other forms of active secondary suspension for guided ground transportation vehicles, although the constraints identified will usually be less critical because the restrictions imposed by the limited airgap with Maglev are quite severe. Nevertheless 'skyhook' or absolute velocity damping is often proposed for active suspensions without consideration of the large deflections it brings about at gradients, and the paper indicates that reconciling this will inevitably mean that the excellent performance which can be shown for the response to random track variations is not achievable.

\section{REFERENCES}

1 Gottzein, E. and Lange, B. Magnetic suspension control systems for the MBB high speed train. Automatica, 1975, 11, 271-284.

2 Jayawant, B. V. Electromagnetic levitation and suspension techniques, 1981 (Edward Arnold, London).

3 Taylor, D. R. D., Goodall, R. M. and Oates, C. D. M. Theoretical and practical considerations in the design of the suspension system for Birmingham Maglev. IMechE Conference on Maglev transport - now and for the future, Solihull, 1984, Paper C414/84, pp. 185-192 (Mechanical Engineering Publications, London).

4 Goodall, R. M. Performance limitations for active secondary railway suspensions. Proceedings of the JSME conference STECH '93, Vol. 2, Yokohama, 1993, pp. 81-86 (Japan Society of Mechanical Engineers, Tokyo).

5 Pollard, M. G. and Williams, R. A. The dynamic behaviour of a low speed electromagnetic suspension. Proceedings of the IUTAM symposium on the Dynamics of vehicles on road and railway tracks, Delft, 1975, pp. 455-479 (Swets and Zeitlinger, Holland).

6 Pollard, M. G. and Simons, N. J. A. Passenger comfort-the role of active suspensions. Proc. Instn Mech. Engrs, Part D, 1984, 198(11), 161-175.

7 Goodall, R. M. and Williams, R. A. Dynamic criteria in the design of Maglev suspension systems. IMechE Conference on Maglev transport-now and for the future, Solihull, 1984, Paper C394/84, pp. 77-85 (Mechanical Engineering Publications, London).

8 Hedrick, J. K. Railway vehicle active suspensions. Veh. Syst. Dyn., 1981, 10, 267-283.

9 Williams, R. A., Best, A. and Crawford, I. L. Refined low frequency active suspension. IMechE Conference on Vehicle ride and handling, Birmingham, 1993, pp. 285-300. (Mechanical Engineering Publications, London).

10 Gottzein, E., Meisinger, R. and Miller, L. The magnetic wheel in the suspension of high speed ground transportation vehicles. IEEE Trans Veh. Technol., 1980, VT-29(1), 17-23. 